\title{
Integrated expression analysis of muscle hypertrophy identifies Asb2 as a negative regulator of muscle mass
}

\author{
Jonathan R. Davey, ${ }^{1}$ Kevin I. Watt, ${ }^{1}$ Benjamin L. Parker, ${ }^{2}$ Rima Chaudhuri, ${ }^{2}$ James G. Ryall, ${ }^{3,4}$ \\ Louise Cunningham, ${ }^{1}$ Hongwei Qian, ${ }^{1}$ Vittorio Sartorelli, ${ }^{3}$ Marco Sandri, ${ }^{5}$ Jeffrey Chamberlain, ${ }^{6}$ \\ David E. James, ${ }^{2,7}$ and Paul Gregorevic ${ }^{1,4,6,8}$ \\ 'Baker IDI Heart and Diabetes Institute, Melbourne, Australia. ${ }^{2}$ Charles Perkins Centre, School of Molecular Bioscience, \\ The University of Sydney, Sydney, Australia. ${ }^{3}$ National Institute of Arthritis Musculoskeletal and Skin Diseases (NIAMS), \\ $\mathrm{NIH}$, Bethesda, Maryland, USA. ${ }^{4}$ Department of Physiology, The University of Melbourne, Melbourne, Australia. \\ ${ }^{5}$ Venetian Institute of Molecular Medicine, The University of Padova, Padova, Italy. ${ }^{6}$ Department of Neurology, \\ The University of Washington, Seattle, Washington, USA. 'Sydney Medical School, The University of Sydney, \\ Sydney, Australia. ${ }^{8}$ Department of Biochemistry and Molecular Biology, Monash University, Clayton, Australia.
}

The transforming growth factor- $\beta$ (TCF- $\beta$ ) signaling network is a critical regulator of skeletal muscle mass and function and, thus, is an attractive therapeutic target for combating muscle disease, but the underlying mechanisms of action remain undetermined. We report that follistatin-based interventions (which modulate TCF- $\beta$ network activity) can promote muscle hypertrophy that ameliorates aging-associated muscle wasting. However, the muscles of old sarcopenic mice demonstrate reduced response to follistatin compared with healthy youngadult musculature. Quantitative proteomic and transcriptomic analyses of young-adult muscles identified a transcription/translation signature elicited by follistatin exposure, which included repression of ankyrin repeat and SOCS box protein 2 (Asb2). Increasing expression of ASB2 reduced muscle mass, thereby demonstrating that $A s b 2$ is a TCF- $\beta$ network-responsive negative regulator of muscle mass. In contrast to young-adult muscles, sarcopenic muscles do not exhibit reduced ASB2 abundance with follistatin exposure. Moreover, preventing repression of ASB2 in young-adult muscles diminished follistatin-induced muscle hypertrophy. These findings provide insight into the program of transcription and translation events governing follistatin-mediated adaptation of skeletal muscle attributes and identify $A s b 2$ as a regulator of muscle mass implicated in the potential mechanistic dysfunction between follistatin-mediated muscle growth in young and old muscles.

Conflict of interest: The authors have declared that no conflict of interest exists.

Submitted: November 9, 2015 Accepted: March 23, 2016 Published: April 21, 2016

Reference information: JCI Insight. 2016;1(5):e85477. doi:10.1172/ji.insight.85477.

\section{Introduction}

Aging beyond young adulthood in humans is accompanied by a progressive loss of skeletal muscle mass and strength, which accelerates with advanced aging $(1,2)$. Diminishing muscle mass and functionality contribute to the manifestation of sarcopenia and severe frailty that directly impacts on physical ability and metabolism, with significant consequences for risk of fall-related injury and the development and/or exacerbation of other medical conditions. Consequently the loss of functional musculature constitutes a leading contributor to increased morbidity and poor long-term prognosis in the elderly aging population. The underlying mechanisms that promote the development and progression of sarcopenia are poorly understood, but contributing factors include diminishing levels of anabolic hormones $(3,4)$, altered ubiquitin-proteasome activity (5), mitochondrial dysfunction (6), and deterioration of the interaction between muscle fibers and motor nerves (7, 8). Currently, interventions that slow age-related muscle wasting have largely been limited to participation in regular resistance exercise (9). Development of drugs as alternative or complementary interventions holds considerable appeal, especially for restoring muscle function in individuals that are already severely frail who may struggle to participate in exercise protocols of sufficient volume and intensity. One strategy for potentially preventing or reversing sarcopenic muscle wasting is to manipulate the transforming growth factor- $\beta$ (TGF- $\beta$ ) signaling network in muscle to shift the processes controlling protein turnover in favor of conservation and/or accretion $(9,10)$. 
Members of the TGF- $\beta$ family of ligands that engage the type IIB activin receptor (ACTR2B) to stimulate signal transduction via SMAD2 and SMAD3 transcription factors are important regulators of skeletal muscle development and remodeling. These proteins include myostatin (or growth differentiation factor 8 , GDF8), activin A and B. Genetic ablation of myostatin promotes greater numbers of muscle fibers being formed during myogenesis and increased growth of muscle fibers during maturation $(11,12)$, whereas inhibition of myostatin in adult musculature stimulates muscle fiber hypertrophy $(13,14)$. Conversely, overexpression of myostatin in mature musculature causes atrophy by shifting protein turnover in favor of catabolism (15). Similarly to myostatin, inhibition of activin A and B in mice can increase muscle mass (16), whereas overexpression causes significant muscle atrophy and a cachectic phenotype (17-19). Moreover, elevated levels of activin A and B have been observed in malignant conditions associated with severe muscle wasting (17-20). Importantly, there exists a positive correlation between serum activin levels with advancing age that includes a notable upward inflection in humans beyond 60 years of age (21). The identification of specific TGF- $\beta$ family members that negatively impact on muscle attributes via ACTR2BSMAD2/3 and that are associated with conditions of frailty has stimulated considerable interest in the development of therapies to target these ligands and the processes they regulate in muscle $(17,22,23)$.

Alongside exploration of ligand- and receptor-specific inhibitors as possible therapeutics, interest has grown in using follistatin-based (FST-based) interventions as modulators of the TGF- $\beta$ network in skeletal muscle (24). FST binds to the ACTR2B interaction site of myostatin and the activins to prevent engagement with ACTR2B and consequently represses downstream SMAD2/3 signaling $(25,26)$. Loss of FST compromises muscle formation during development $(27,28)$, whereas transgenic or postnatal overexpression of FST generates profound muscle hypertrophy $(12,29)$. Importantly, FST promotes bone morphogenic protein (BMP) signaling in skeletal muscle via the SMAD1/5/8 transcription factors; the signaling was recently identified as a mechanism that positively regulates muscle mass $(30,31)$. As the BMPs are TGF- $\beta$ family members that act on SMAD1/5/8 in parallel with the myostatin/activin/-SMAD2/3 axis, these and complementary findings have revealed that the TGF- $\beta$ network comprises components that act in opposition as positive and negative regulators of muscle mass. Despite the growing appreciation for the interplay between components of the TGF- $\beta$ network to control muscle attributes, the program of transcriptional and translational changes driven by these events to achieve muscle hypertrophy remain undefined.

Manipulation of the TGF- $\beta$ network may hold the potential to address muscle wasting associated with a variety of conditions and with advancing age. We sought to evaluate the impact of FST treatment in age-related muscle wasting. We report here that exposure of muscles to increased levels of FST can confer positive effects on muscle mass and strength in the setting of age-related muscle wasting. However, the hypertrophic effects of FST are diminished in aged muscles relative to young-adult muscles. This discrepancy highlights the importance of revealing the mechanisms of FST-mediated muscle adaptation. To this end, we combined quantitative analyses of proteomic and transcriptomic responses in muscles exposed to FST to identify a transcriptomic and proteomic signature associated with FST-mediated skeletal muscle growth. Combined interrogation of proteomics and transcriptomics revealed that downregulation of ankyrin repeat-containing protein with a suppressor of cytokine signaling box 2 (Asb2), an E3 ubiquitin protein ligase, is a feature of FST-induced muscle hypertrophy and SMAD2/3 control of muscle mass. In aged mice treated with FST, Asb2 repression is diminished, thereby highlighting a discrepancy between the expression of FST and consequent effects in young-adult versus old muscles. ASB2 overexpression in skeletal muscle causes a reduction in muscle fiber size and blunts FST-induced muscle hypertrophy. These findings demonstrate a mechanism for regulation of FST-induced muscle hypertrophy by $A s b 2$ and a new role of $A s b 2$ as a regulator of muscle mass.

\section{Results}

FST-mediated muscle hypertrophy is blunted in sarcopenic mice. We and others have shown that myostatin and activins are negative regulators of muscle mass, such that overexpression causes atrophy, whereas inhibition increases mass. We sought to assess the effect of inhibiting myostatin/activin in muscles demonstrating features of sarcopenia via interventions that promote the expression of FST. To accomplish this, we utilized recombinant adeno-associated virus-based vectors (rAAV vectors) to transduce mouse skeletal muscle with FST-expressing constructs (32). We injected tibialis anterior (TA) muscles of 6- or 24-month-old mice with either rAAV encoding FST (rAAV:FST) or an empty vector control construct (rAAV:MCS) in the muscle of the contralateral leg (Figure 1A). The TA muscles of the 24-month-old mice were approximately $14 \%$ lighter than the 6-month-old mice indicating age-related muscle wasting. FST treatment induced mus- 
A

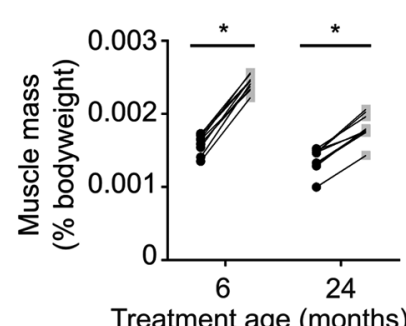

Treatment age (months)

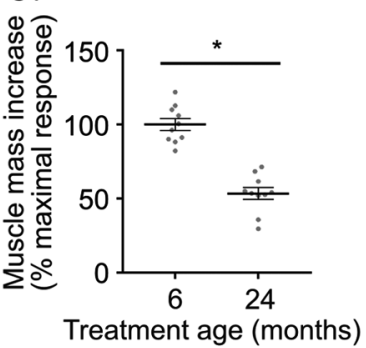

Treatment age (months)
B

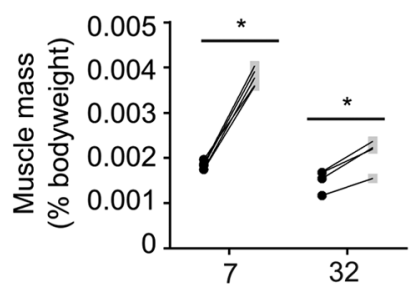

Treatment age (months)
rAAV:MCS $\square$ rAAV:FST

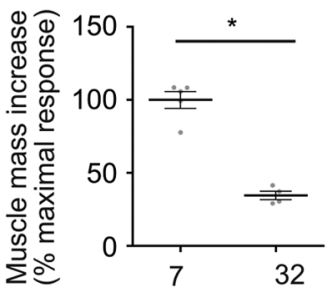

Treatment age (months)
C

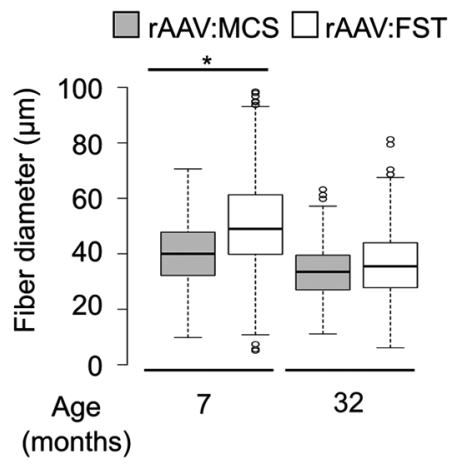

$\mathbf{F}$

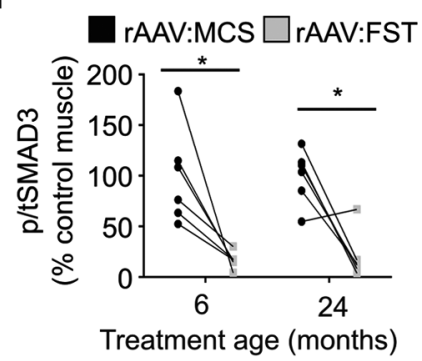

D

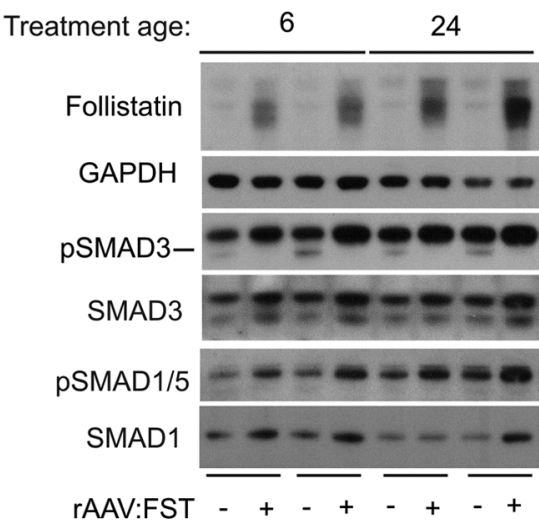

G

rAAV:MCS $\square$ rAAV:FST

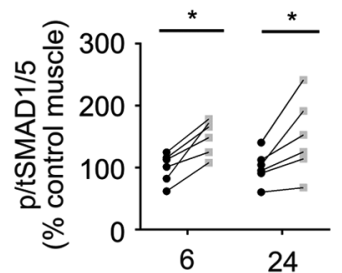

Treatment age (months)
E

rAAV:MCS $\square$ rAAV:FST

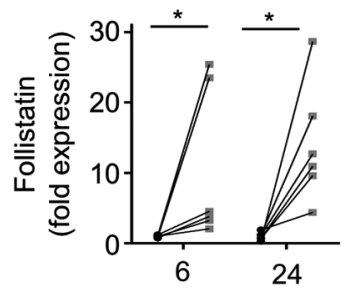

Treatment age (months)

Figure 1. Follistatin gene delivery promotes muscle hypertrophy, which is diminished in old mice. C57BI/6 mice of indicated age were injected with rAAV:MCS in the left TA and rAAV:FST in the right TA. (A) The mass of TA muscles from rAAV:FST-injected mice were compared with the contralateral rAAV:MCS control (ratio-paired $t$ test, $P<0.05, n=10, \pm \mathrm{SEM}$ ). The change in muscle mass between control and follistatin treatment is presented as a percentage of maximal response in 6-month-old mice (unpaired $t$ test, $P<0.05, n=10, \pm \mathrm{SEM}$ ) (B) The muscle weights and response to follistatin treatment as presented in $\mathbf{A}$ for 7 - and 32-month-old mice (ratio-paired $t$ test; $P<0.05 ; 7$ month, $n=5 ; 32$ month, $n=4 ; \pm$ SEM). Maximal response is defined by the follistatin response in 7-month-old mice (unpaired $t$ test; $P<0.05 ; 7$ month, $n=5 ; 32$ month, $n=4 ; \pm \mathrm{SEM}$ ). (C) The diameter of TA muscle fibers from mice reported in B (Holm-Sidak multiple $t$ test; $P<0.05 ; 7$ month, $n=5$; 32 month, $n=4$; \pm SEM). (D) Immunoblot analysis of mice from $\mathbf{A}$ for detection of follistatin expression and SMAD signaling. Quantification of immunoblot analysis from $\mathbf{D}$ for (E) follistatin expression (ratio-paired $t$ test, $P<0.05, n=6, \pm$ SEM). (F) Ratio of phosphorylated to total SMAD3 (ratio-paired $t$ test, $P<0.05, n=6, \pm$ SEM), and (C) ratio of phosphorylated to total SMAD1/5 (ratio -paired $t$ test, $P<0.05, n=6, \pm \mathrm{SEM}$ ). (H) Time course analysis of follistatin expression in TA muscles injected with rAAV:indFST. DOX was administered in food ad libitum for the indicated number of days. $n=2$. (I) The mass of TA muscles from rAAV:indFST-injected mice were compared with the contralateral rAAV:MCS control (ratio-paired $t$ test; $P<0.05$; no DOX, $n=9 ; 2$ days DOX, $n=7 ; 28$ days DOX, $n=12$; \pm SEM). Recombinant adeno-associated virus, rAAV; control construct, MCS; follistatin, FST; tibialis anterior muscle, TA; doxycycline, DOX; tetracycline responsive follistatin, indFST.

cle mass growth in both groups; however, the response was reduced by approximately $40 \%-50 \%$ in the 24-month-old mice. The reduction in muscle growth was more severe in extremely old mice. FST treatment of the TA muscle in 7- and 32-month-old mice with rAAV:FST resulted in an approximately 70\% reduction in muscle hypertrophy (Figure 1B). Consistent with the diminished hypertrophic response of treated muscles in old mice, histological examination demonstrated that muscle fiber diameter was increased as a consequence of treatment in young-adult mice, whereas effects were reduced in treated old muscles (Figure 1C). In 7-month-old mice, rAAV:FST treatment increased the fiber diameter by approximately $25 \%-30 \%$ across all interquartile ranges compared with rAAV:MCS-treated muscles. In the 32-month-old mice, we found no difference in the fiber diameter interquartile ranges of TA muscles treated with rAAV:FST ver- 
A rAAV:TetON
rAAV:indFST

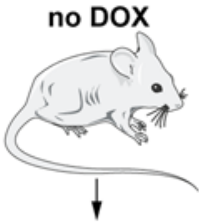

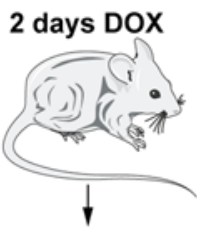

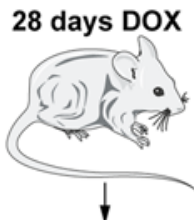

dissect muscle, extract protein, digest with LysC / trypsin multiplex stable isotope labelling with ITRAQ

114 or 117

115 or 116

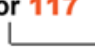

pool and fractionate with TSK-Amide-HILIC HPLC

idwitwithit

nanoUHPLC-MS/MS (Q-Exactive)

\section{B}

5,375 proteins quantified ( 2 out 3 replicates)

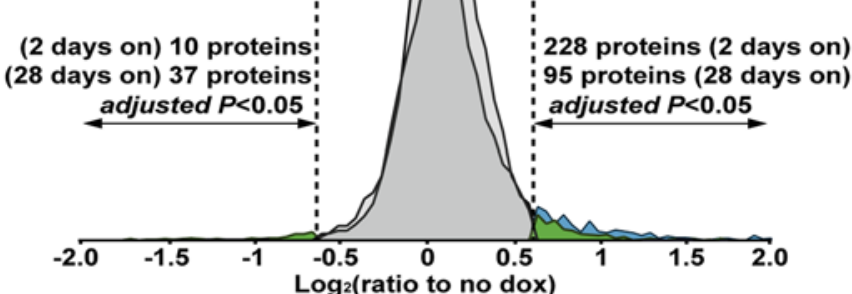

$\mathbf{E}$

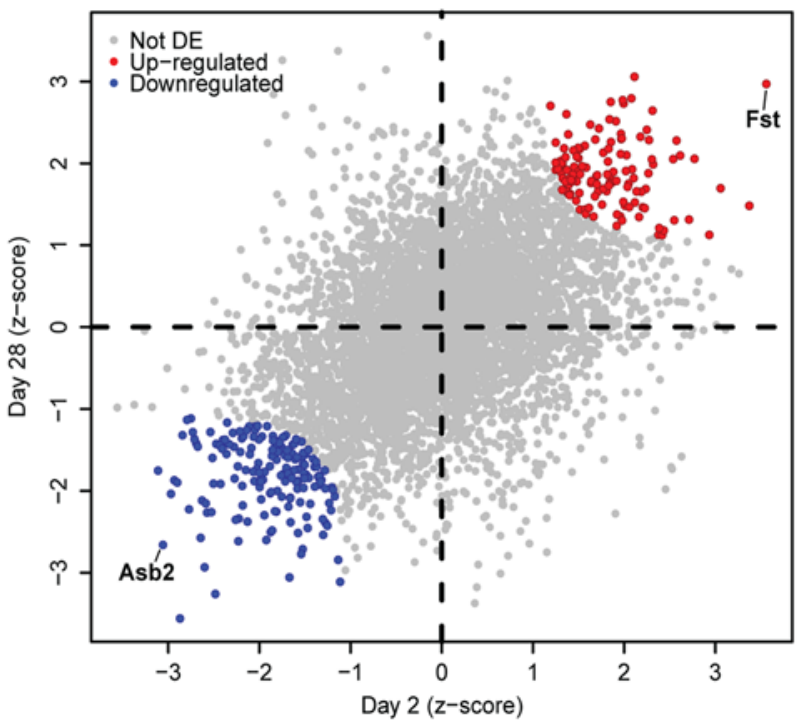

C

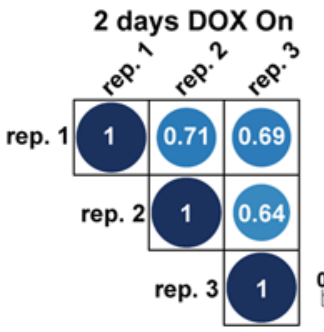

28 days DOX On

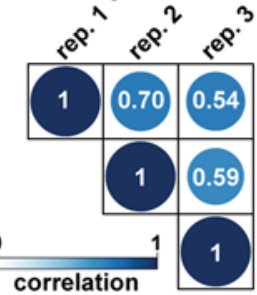

D

Glycosaminoglycan Degradation Fatty Acid Metabolism Glycosphingolipid Biosynthesis Globo Series Drug Metabolism And Other Enzymes -

Chemokine Signaling Pathway Notch Signaling Pathway Cell Adhesion Molecules Adherens Junction Complement And Coagulation Cascades Nod-like Receptor Signaling Pathway Cytosolic DNA Sensing Pathway Cysteine And Methionine Metabolism -

Fructose And Mannose Metabolism Glutathione Metabolism Starch And Sucrose Metabolism Inositol Phosphate Metabolism Glyoxylate And Dicaryruvate Metabolism Folate Biosynthesis Sulfur Metabolism Metabolism Of Xenobiotics By Cytochrome P450 Drug Metabolism Cytochrome P450 Erbb Signaling Pathway Wnt Signaling Pathway Renin Angiotensin System Insulin Signaling Pathway Aminoacyl Trna Biosynthesis = TCA cycle Oxidative Phosphorylation Valine, Leucine And Isoleucine Degradation Calcium Signaling Pathway Cardiac Muscle Ontraction Vascular Smooth Muscle Contraction Ecm Receptor Interaction Hypertrophic Gnrh Signaling Pathway Arrhythmogenic Right Ventricular Cardiomyopathy = Dilated Cardiomyopathy Protein Digestion And Absorption Lysosome
Snare Interactions In Vesicular Transport
Endocytosis
Antigen Processing Ann Presentation Antigen Processing And Presentation Jak-Stat Signaling Pathway Regulation Of Actin Cytoskeleton Amino Sugar And Nucleotide Sugar Metabolism Steroid Biosynthesis Proteasome Axon Guidance Arginine And Proline Metabolism -
Toll-like Receptor Signaling Pathway Tryptophan Metabolism Purine Metabolism Propanoate Metabolism Riboflavin Metabolism RNA Degradation -
PPAR Signaling Pathway

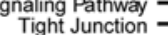

Proximal Tubule Bicarbonate Reclamation MAPK Signaling Pathway Pentose Phosphate Pathway Neurotrophin Signaling Pathway -

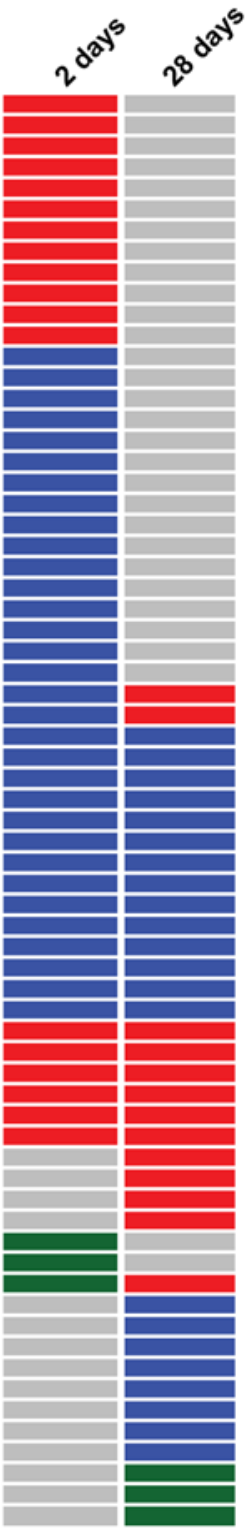

Figure 2. Quantitative proteomic analysis reveals changes in protein signature associated with acute and chronic follistatin expression in skeletal muscle. (A) Schematic displaying the experimental workflow for iTRAQ sample labeling and tandem mass spectrometry (MS/MS) analysis. (B) Distribution of the quantified proteins relative to no-DOX samples with a \pm 1 .5-fold expression change cut-off (moderated $t$ test, adjusted $P<0.05, n=3$ ). (C) Pearson correlation between replicates from 2- and 28-day follistatin treatments. (D) Gene set enrichment analysis predicted pathways regulated across the indicated time points in the proteomics data set. The upregulated pathways are shown in red, downregulated are shown in blue, and pathways showing a mixed response are highlighted in green $(P<0.05)$. (E) Z-score scatterplot of the proteins concordantly changed at 2 and 28 days from the 2 -dimensional directional pathway analysis. Significantly upregulated proteins are highlighted in red in quadrant I, and downregulated proteins are cultured in blue in quadrant III (integrated $P<0.05$ ); proteins that remained unaltered under the tested hypotheses are shown in gray. Isobaric labeling for relative and absolute quantitation, iTRAQ; hydrophilic interaction chromatography, HILIC; nano-ultra high performance liquid chromatography, nanoUHPLC; differentially expressed, DE; recombinant adeno-associated virus, rAAV; tibialis anterior muscle,TA; doxycycline, DOX; tetracycline responsive follistatin, indFST. 
sus rAAV:MCS. These changes in fiber diameter were largely conserved across the distribution of muscle fibers; however, a trend for increased fiber diameter was observed in rAAV:FST-treated old muscles (Supplemental Figure 1, A and B; supplemental material available online with this article; doi:10.1172/jci. insight.85477DS1).

Circulating levels of myostatin, activin, and other TGF- $\beta$ family members that promote deleterious SMAD2/3 signaling in muscle have been shown to change with age, which could potentially impact on the effect of FST in old muscles. To determine whether the differences in FST-mediated effects between youngadult and old mice were a consequence of differential FST expression or effects upon the SMAD signaling, we examined FST abundance and SMAD phosphorylation in response to rAAV:FST administration between the age groups. We found that expression of FST was not different between the treated muscles of young-adult and old mice (Figure $1, \mathrm{D}$ and $\mathrm{E}$ ) and that treated muscles exhibited comparably reduced SMAD2/3 phosphorylation (Figure 1, D and F) and increased SMAD1/5 phosphorylation (Figure 1, D and G). As FST gene delivery has been shown capable of completely inhibiting muscle wasting and signaling changes caused by supraphysiological expression of myostatin and activin that vastly exceeds the levels experienced with aging $(18,21,32)$, these findings demonstrate that the reduced hypertrophic effect of rAAV:FST administration to old muscles is not a consequence of reduced FST expression or compromised modulation of SMAD signaling $(18,21)$. These data indicate that, while FST-mediated inhibition of myostatin/activin signaling elicits muscle hypertrophy in aged muscle, the response to this intervention is diminished compared with young mice. To identify mechanistic discrepancies between these settings of muscle hypertrophy, we sought to elucidate the proteomic and transcriptomic adaptations that occur in muscles as a consequence of FST treatment.

Development of $r A A V$ vectors to achieve inducible FST expression in muscle. We sought to define the impact of FST treatment upon the skeletal muscle proteome and transcriptome and to stratify effects into acute and chronic responses. To achieve this objective, we used rAAV vectors to transduce mouse muscles with constructs designed to provide tetracycline-controlled activation of transcription (16). We injected the TA muscles of mice with rAAV vectors carrying a tetracylcine-inducible gene expression construct (TetOn) construct and a TetOn-responsive FST construct (rAAV:TetOn and rAAV:indFST, respectively) or a control construct (rAAV:TetOn and an empty vector, rAAV:MCS) in the muscle of the contralateral limb. Seven days after injection, mice were administered doxycycline (DOX, as DOX-supplemented chow) to induce FST expression for a given number of days. FST expression was apparent after 2 days of DOX feeding and strongly detectable after 28 days of DOX administration (Figure 1H). In contrast, FST was not observed in mice administered rAAV:TetOn and rAAV:indFST without DOX.

To determine the acute and chronic response to FST expression in skeletal muscle, we treated mice with rAAV:indFST and DOX for 2 and 28 days, respectively. Mice received rAAV:TetOn plus rAAV:indFST in the treated TA muscle and rAAV:TetOn plus control vector in the contralateral TA muscle. Treated muscles in which FST expression was induced for 28 days demonstrated a profound hypertrophy amounting to an average doubling of mass by 4 weeks after induction (Figure 1I). Mice administered vectors but not DOX were observed for 5 weeks after injection and showed no detectable change in muscle mass. Muscles examined after as little as 48 hours of DOX feeding exhibited evidence of increased mass, demonstrating that FST-mediated changes in the muscle proteome/transcriptome that occur within as little as 2 days are sufficient to promote growth and constitute an acute response to myostatin/activin inhibition.

The proteomic and transcriptomic signature of FST-induced muscle hypertrophy. To identify changes in protein expression during acute and chronic FST treatment, we employed a quantitative proteomic analysis of muscles using isobaric tags for relative and absolute quantification (iTRAQ) (Figure 2A). We compared the proteomes of the rAAV:indFST injected muscles from (i) no DOX control, (ii) 2 days DOX administration, and (iii) 28 days DOX administration. A total of 5,375 proteins were quantified in 2 of 3 biological replicates (Figure 2B and Supplemental Table 1). Muscles injected with rAAV:indFST from the 2- and 28-day DOX treatment groups demonstrated upregulation of 228 and 95 proteins, relative to the no DOX control group (1.5-fold cut-off, $t$ test, adjusted $P<0.05)$. rAAV:indFST-injected muscles across the 2 - and 28-day DOX treatments exhibited downregulation of 10 and 37 proteins, relative to the no DOX control. Good correlation was observed between the 3 biological replicates with an average Pearson correlation of 0.69 and 0.61 between replicates from 2- and 28-day DOX treatments relative to no DOX control, respectively (Figure $2 \mathrm{C}$ ). These data demonstrate that FST overexpression in mouse muscles causes rapid changes in protein abundance. 


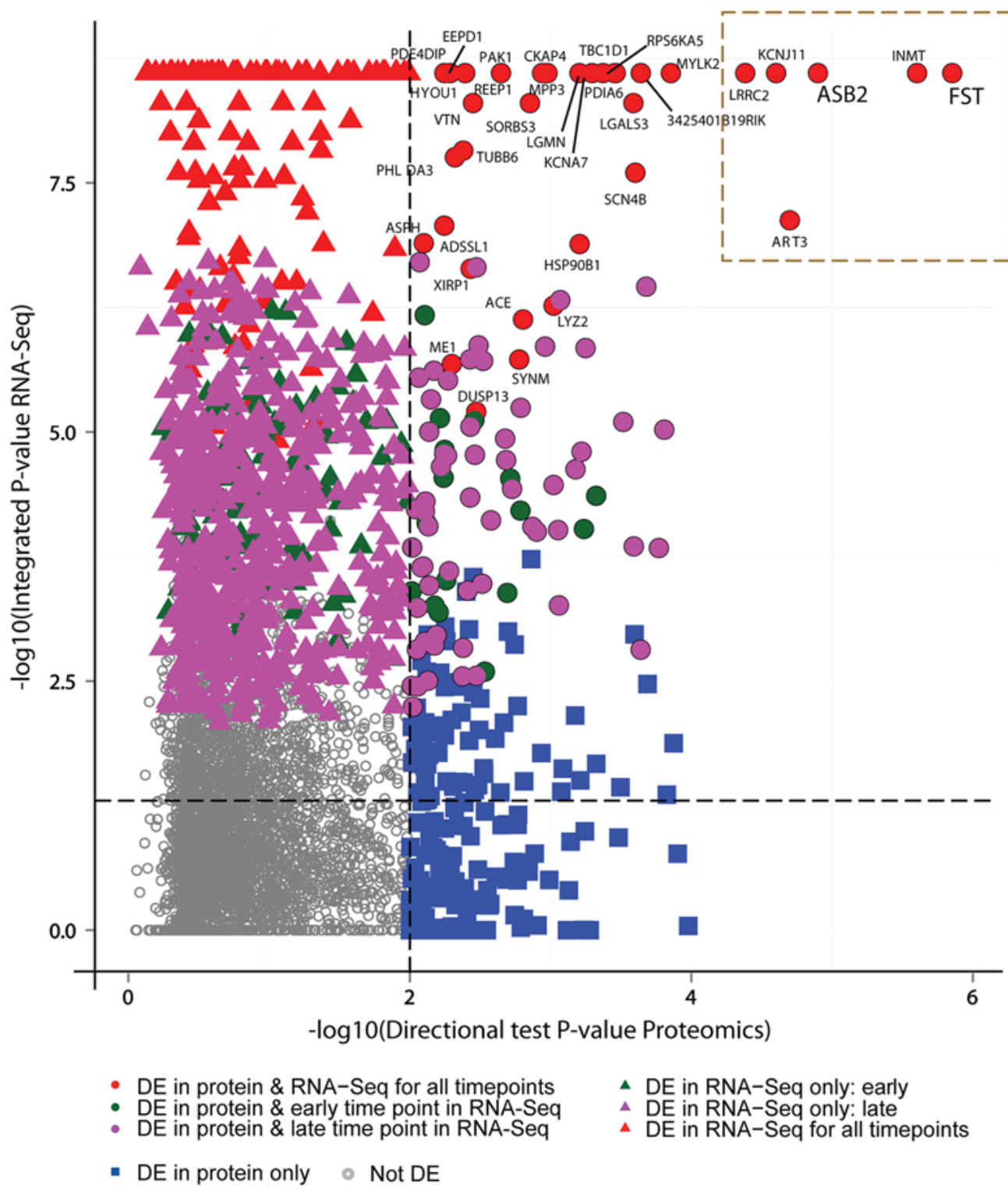

Figure 3. Differential expression of sequenced transcripts and proteins in response to acute and chronic follistatin expression in skeletal muscle. Overlap between significant differentially expressed (DE) proteins and genes from the proteomic and RNA-Seq data sets were categorized into 8 groups. Shapes are used to annotate changes specific to each data set. Proteins and genes significantly altered in both the proteome and transcriptome analysis are represented in circles, transcript-only changes are represented by triangles, and protein-only changes are represented by squares. Colors are used to code for the temporal factor; changes across all time points are shown in red, acute transcript changes are shown in green, and chronic transcript changes are shown in magenta. Significantly changed proteins during acute and chronic treatment without any transcript level change observed are shown in blue. Unchanged genes/proteins are shown in gray unfilled circles. Significance of each protein/gene was determined from the bioinformatics analysis in their respective data sets.

To obtain an overview of the regulated pathways at the proteome level, we performed a gene set enrichment analysis (GSEA; http://software.broadinstitute.org/gsea/msigdb) from the Molecular Signature Database Collection and Kyoto Encyclopedia of Genes and Genomes (KEGG) (Figure 2D). Numerous pathways involved in catabolic and metabolic processes were regulated between the muscles exposed to FST, relative to no DOX control. These analyses illustrate the complexity of protein adaptations driving muscle hypertrophy as a consequence of FST exposure. We next compared the abundance of proteins between the muscles from the 2- and 28-day treatments to investigate sustained up- or downregulation of specific proteins with ongoing FST expression (via continued DOX administration). We performed 2-dimensional hypothesis testing using directional pathway analysis (DPA) and a 1-sided version of Pearson's method (OSP) for combining $P$ values across the 2 time points. A total of 296 proteins were identified, 
including 127 and 169 up- and downregulated proteins for the 2- and 28-day DOX treatments (Z-score adjusted $P<0.05$; Figure $2 \mathrm{E}$ and Supplemental Table 2). Validating our approach, we observed that FST was the most significantly upregulated protein after both 2 days and 28 days of DOX administration.

To investigate which FST-induced changes in protein abundance were associated with altered gene expression, we quantified the transcriptome of muscles by RNA-sequencing (RNA-Seq). These analyses examined samples of mouse TA muscles previously collected after acute expression of FST (3 days) or chronic expression ( 7 and 14 days). An average of 24,359 gene products were mapped across each time point, with 253 and 1,501 gene products significantly regulated during the acute and chronic treatments, respectively, relative to $\mathrm{rAAV}: \mathrm{MCS}$ injected control TA muscles $(t$ test adjusted $P<0.05, \pm 1.5$-fold, fragments per kilobase of transcript per million mapped reads (FPKM) $>1$; Supplemental Table 3). Differential expression of 162 genes was shared between the acute and chronic time point.

We subsequently interrogated the proteome and transcriptome jointly and calculated the overlap between the proteins that changed significantly in the proteome (at 2 and/or 28 days of FST expression) and the genes that were significantly altered in the RNA-Seq data at acute and/or chronic time points. This approach allowed a pair-wise comparison with the proteomics data: (i) acute time point from the RNA-Seq data was compared with the 2 days DOX proteomics data, and (ii) chronic time point from the RNA-Seq data was compared with the 28 days DOX proteomics data. A total of 3,716 gene products were quantified in both the RNA-Seq and proteomics data. We classified the genes under the following 3 categories: (i) significantly altered at all time-points in the transcriptome and proteome: 35 genes; (ii) altered in both proteome and transcriptome but only in the early time point in RNA-Seq data: 16 genes; and (iii) altered in both data sets but only late in the RNA-Seq data: 60 genes (Figure 3 and Supplemental Table 4). We also calculated genes that were significantly altered only in the transcriptome and not in the proteome and vice versa. There were 115 genes that changed across time points in the transcriptome but not in the proteome. A large number of acute and chronic transcriptome changes (111 and 524 genes, respectively) were not accompanied by a change in the proteome. By proteome analysis, we observed 170 gene products that were differentially expressed without concomitant transcriptome alterations. Consistent with the proteomics data, the FST gene product was the most significantly upregulated transcript in the RNA-Seq data. Based on this experimental configuration, we cannot rule out that a subset of differentially expressed proteins observed in the proteomic data may be due to administration of DOX. However, any such potential changes would be restricted to the proteome-only data set.

Integrated proteome/transcriptome interrogation identifies ASB2 as a FST-responsive gene. The ankyrin repeat-containing protein with a suppressor of $A s b 2$ is a specificity subunit of the Elongin C-Cullin-SOCS box (ECS) type E3 ubiquitin ligase complex $(33,34)$, which we identified as one of the most responsive candidates in the proteomic and RNA-Seq analysis (Figure 2D, Figure 3, and Supplemental Tables 1-4). $A s b 2$ regulates actin cytoskeleton dynamics in hematopoietic cells and is implicated in the regulation of myogenic differentiation $(35,36)$. We observed a 2-fold suppression of ASB2 expression after 2 and 28 days of DOX-induced FST expression. Based on this observation, we sought to test whether suppression of ASB2 is a requirement for this mode of muscle growth. We profiled the expression of ASB2 in response to FST to validate the proteomic data. Immunoblot and quantitative PCR (qPCR) analysis of Asb2 expression in AAV:indFST-treated muscles showed an approximately $45 \%$ and $60 \%$ suppression, respectively, with 2 days of DOX administration (Figure 4, A-C) that was sustained throughout DOX treatment. There was no difference in ASB2 levels in muscles receiving rAAV:indFST but no DOX. These data indicate that suppression of $A s b 2$ transcription and protein expression is an early feature of FST expression in skeletal muscle.

As FST-driven muscle hypertrophy is the product of signaling changes through SMAD2/3 and SMAD1/5/8 pathways, we examined whether control of ASB2 expression could be independently attributed to either pathway. We tested activation of the SMAD1/5/8 pathway through the delivery of a rAAV vector encoding a constitutively active mutant of the ALK3 receptor (rAAV:caALK3), which increases phosphorylation of SMAD1/5/8 and results in muscle hypertrophy (31). The TA muscles of C57BL/6 mice were injected with $\mathrm{rAAV}: \mathrm{caALK} 3$ in one leg and received $\mathrm{rAAV}: \mathrm{MCS}$ in the contralateral muscle. Two weeks after injection, no differences in ASB2 levels were observed between treatments (Supplemental Figure 2A). We subsequently tested the contribution of the SMAD2/3 pathway as a regulator of ASB2 through the delivery of an AAV vector encoding SMAD7 (rAAV:SMAD7). SMAD7 is an inhibitory SMAD protein that blocks phosphorylation of SMAD2/3 by competitive binding to the type-I activin 
A

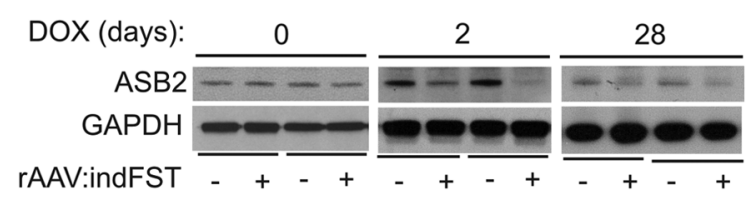

B

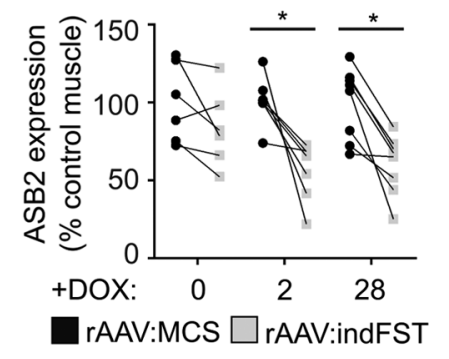

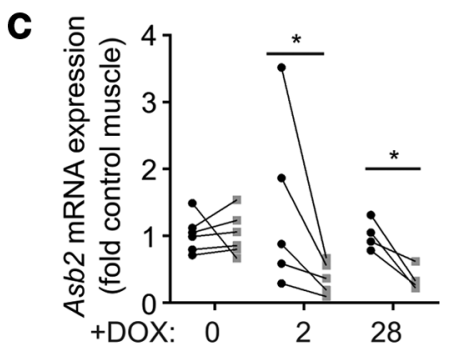

rAAV:MCS $\square$ rAAV:indFST
D

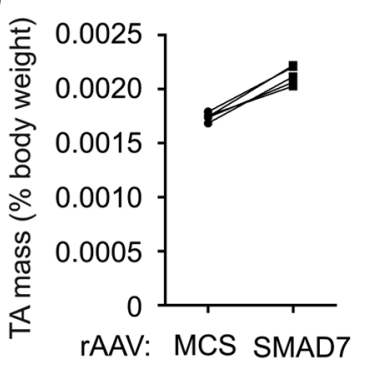

E

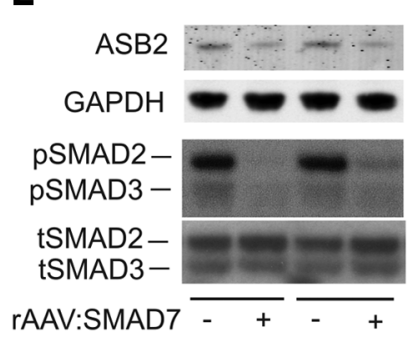

$\mathbf{F}$

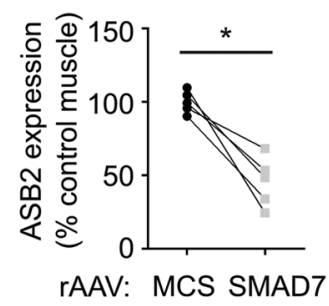

G

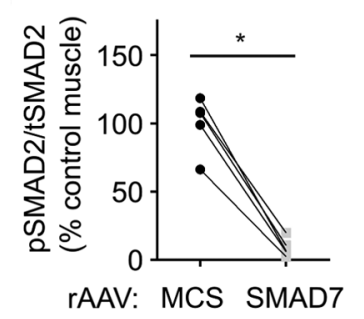

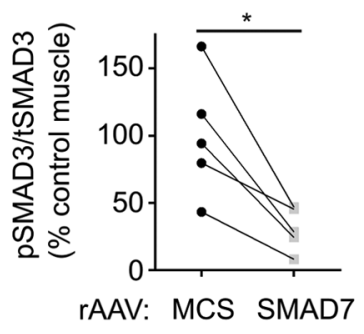

$\mathbf{H}$

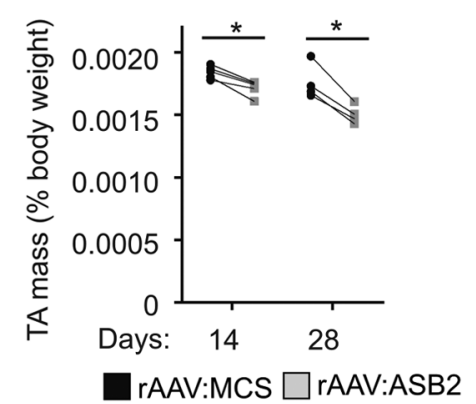

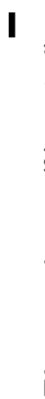

Figure 4. Asb2 is an atrogene that is downregulated during follistatin treatment. (A) C57BI/6 mice were injected with rAAV:MCS in the left TA and rAAV:indFST in the right TA. Immunoblot analysis of ASB2 expression in response to indicated follistatin treatments. (B) Quantification of ASB2 expression presented in A (ratio-paired $t$-test; $P<0.05$; no DOX, $n=6$; 2 days DOX, $n=7 ; 28$ days DOX, $n=8 ; \pm \mathrm{SEM}$ ). (C) Quantitative PCR analysis of $A$ sb2 mRNA in response to indicated treatments (ratio-paired $t$-test; $P<0.05 ;$ no DOX, $n=6 ; 2$ days DOX, $n=5 ; 28$ days DOX, $n=4 ; \pm$ SEM). (D) The mass of TA muscles of C57BI/6 mice after injection of rAAV:MCS in the left TA and rAAV:SMAD7 in the right TA. Mass values were compared between contralateral TA muscles from individual mice (ratio-paired $t$-test, $P<0.05, n=5$, \pm SEM). (E) Immunoblot analysis of mice from $\mathbf{D}$ for ASB2 expression and SMAD signaling. Quantification of immunoblots from $\mathbf{E}$ for $(\mathbf{F})$ ASB2 expression (ratio-paired $t$ test, $P<0.05, n=5, \pm$ SEM) and (C) ratio of phosphorylated to total SMAD3 and ratio of phosphorylated to total SMAD2 (ratio-paired $t$ test, $P<0.05, n=5, \pm \mathrm{SEM}$ ). (H) C57BI/ 6 mice were injected with rAAV:MCS in the left TA and rAAV:Asb2 in the right TA. The mass of TA muscles from rAAV:Asb2-injected muscles were compared with contralateral control muscles (ratio-paired $t$ test; $P<0.05 ; 14$ days, $n=5 ; 28$ days, $n=4$; \pm SEM). (I) Change in muscle mass between rAAV:Asb2- and control-treated TA muscles from $\mathbf{H}$ at indicated time points (unpaired $t$ test; $P<0.05 ; 14$ days, $n=5$; 28 days, $n=4$; \pm SEM). Recombinant adeno-associated virus, rAAV; ankyrin repeat-containing protein with a suppressor of cytokine signaling box 2, Asb2; control construct, MCS; follistatin, FST; tibialis anterior muscle, TA; doxycycline, DOX; tetracycline responsive follistatin, indFST.

receptor and subsequent receptor degradation $(37,38)$. Two weeks after administering rAAV:SMAD7 to the TA muscles of C57BL/6 mice, muscle mass compared with the control rAAV:MCS was increased by approximately $25 \%$ (Figure 4D). The expression of ASB2 was found to be reduced by approximately $50 \%$ in AAV:SMAD7-treated muscles, and the phosphorylation of SMAD2 and SMAD3 was potently reduced (Figure 4, E-G). Collectively, these results indicate that ASB2 expression correlates to changes in signaling through the SMAD2/3 pathway in muscle.

ASB2 expression negatively regulates skeletal muscle mass. Reasoning that Asb2 may operate as a regulator of muscle mass, we investigated the consequences of $A s b 2$ overexpression on muscle mass homeostasis. To this end, we generated an rAAV vector encoding the muscle-specific isoform of $A s b 2$ (Asb2 $\beta$ ) (35) with an N-terminal FLAG-tag (rAAV:Asb2) (Supplemental Figure 2B). The TA muscles of C57B1/6 mice were injected with rAAV:Asb2, and the contralateral TA muscle received an injection of rAAV:MCS control vector. The TA muscles that received rAAV:Asb2 were reduced in mass by approximately $7 \%$ at 2 weeks 
A

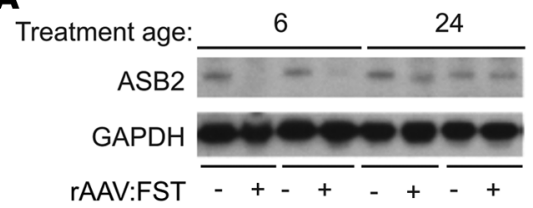

D

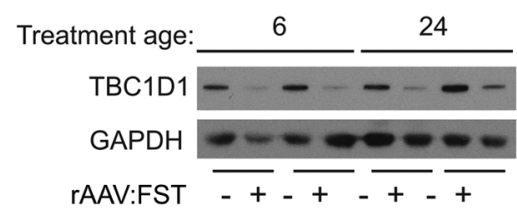

B

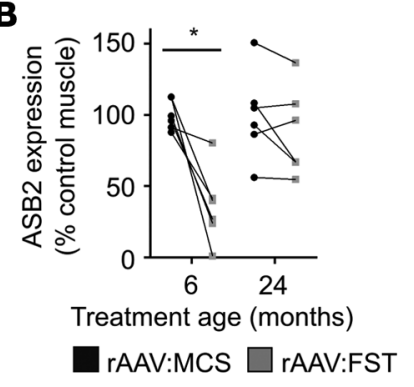

E

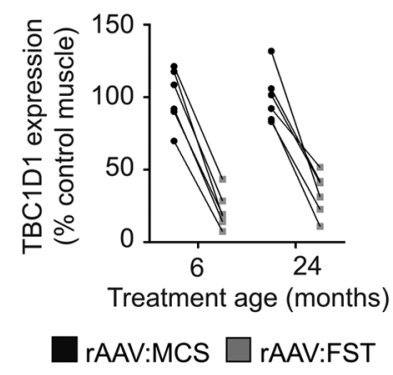

C

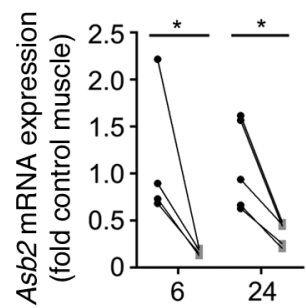

Treatment age (months)

rAAV:MCS $\square$ rAAV:FST

$F$

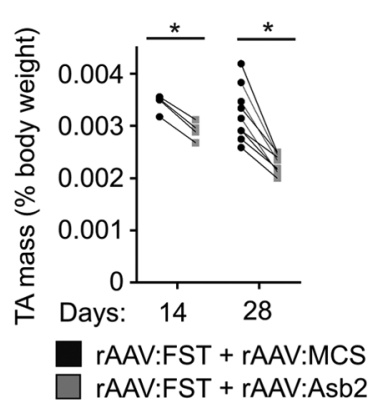

G

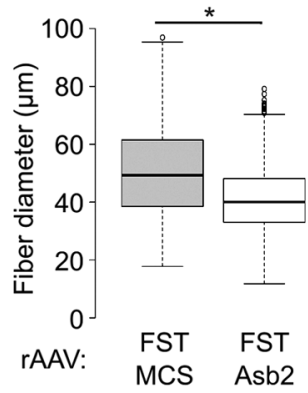

H

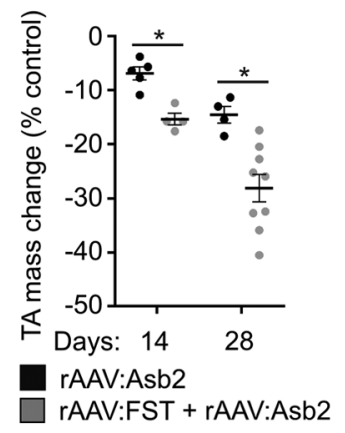

I

Days post-surgery:

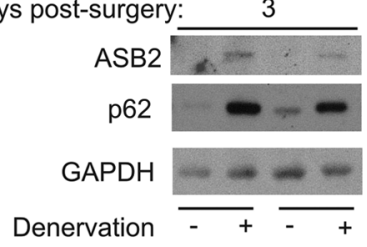

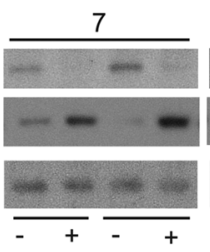

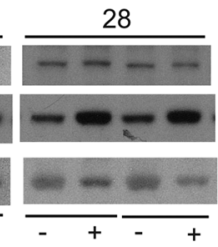

J

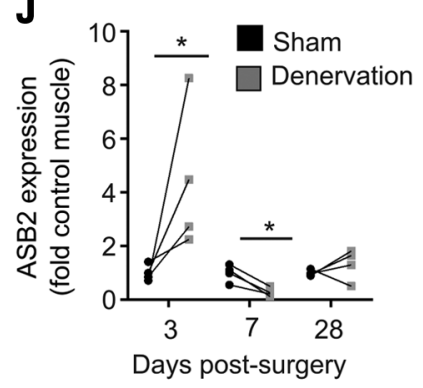

Figure 5. ASB2 is not downregulated in aged muscle exposed to follistatin, but suppression is required for maximal follistatin response. C57BI/6 mice of indicated age were injected with rAAV:MCS in the left TA and rAAV:FST in the right TA. (A) Immunoblot analysis of ASB2 expression in response to indicated follistatin treatment. (B) Quantification of ASB2 expression presented in A (ratio-paired $t$ test, $P<0.05, n=6, \pm S E M$ ). (C) Quantitative PCR analysis of $A s b 2$ mRNA from mice in $\mathbf{A}$ (ratio-paired $t$ test; $P<0.05 ; 6$ month, $n=4 ; 24$ month, $n=5 ; \pm$ SEM). (D) Immunoblot analysis of TBC1D1 expression in response to indicated follistatin treatment. (E) Quantification of TBC1D1 expression presented in $\mathbf{D}$ (ratio-paired $t$ test, $P<0.05, n=$ 6, \pm SEM). (F) C57BI/6 mice were injected with rAAV:FST plus rAAV:MCS in the left TA and rAAV:FST plus rAAV:Asb2 in the right TA. The rAAV:FST plus rAAV:Asb2-coinjected muscles were compared against the rAAV:FST plus rAAV:MCS control muscles (ratio paired $t$ test; $P<0.05 ; 14$ days, $n=$ 4; 28 days, $n=8 ; \pm$ SEM). (G) Muscle fiber diameter in TA muscles examined 28 days after treatment with rAAV:FST plus rAAV:MCS or rAAV:FST plus rAAV:Asb2 (Holm-Sidak multiple $t$ test, $P<0.05, n=4, \pm \mathrm{SEM}$ ). (H) Comparison in relative changes in muscle mass due to Asb2 expression in static (Figure 4E) versus follistatin (F) treatment (unpaired $t$ test; $P<0.05$; rAAV:Asb2 14 days, $n=5$; rAAV:FST + rAAV:Asb2 14 days, $n=4$; rAAV:Asb2 28 days, $n=4$; rAAV:FST + rAAV:Asb2 28 days, $n=8$; \pm SEM). (I) C57BI/6 mice underwent surgical denervation or sham operation on contralateral TA muscles. Immunoblot analysis of indicated time course after surgery for ASB2 expression. (J) Quantification of immunoblots from I for ASB2 expression (ratio-paired $t$ test, $P<0.05, n=4, \pm \mathrm{SEM}$ ). Recombinant adeno-associated virus, rAAV; ankyrin repeat-containing protein with a suppressor of cytokine signaling box 2, Asb2; control construct, MCS; follistatin, FST; tibialis anterior muscle, TA; doxycycline, DOX; tetracycline responsive follistatin, indFST; TBC1 domain family member 1, TBC1D1.

and by approximately $15 \%$ at 4 weeks after injection (Figure $4 \mathrm{H}$ ). These results demonstrate that ASB2 abundance contributes to determining muscle mass in the basal state and that increasing ASB2 expression drives a progressive atrophic response (Figure 4I).

ASB2 is repressed to achieve FST-induced muscle hypertrophy and is refractory to FST treatment in aged muscle. We observed that ASB2 suppression was a feature of myostatin/activin inhibition by FST and sought to determine whether this response was preserved in elderly mice (Figure 1A). Immunoblot analysis of the 6-month-old mice treated with rAAV:FST demonstrated an approximately $60 \%$ reduction of ASB2 expression compared with the control muscle (Figure 5A). However, repression of ASB2 by FST treatment was ameliorated in 24-month-old mice, presenting as a modest trend that did not reach statistical significance 
compared with the control muscle (Figure 5B). ASB2 abundance was not found to be significantly different between the untreated muscles of old mice versus young-adult mice; however, there was some evidence of increased ASB2 levels in the untreated muscles of a subset of the old mice (Supplemental Figure 2C). As $A s b 2$ mRNA transcript was downregulated in response to FST treatment (Figure 4C and Supplemental Table 3), we sought to determine if this repression was maintained in the 24-month cohort. Analysis by qPCR showed that suppression of $A s b 2$ transcript is maintained across both age groups (Figure $5 \mathrm{C}$ ). We analyzed the expression of another gene from the combined data set to determine whether this disparity was specific for $A s b 2$ or common to other FST-responsive genes. We found the TBC1 domain family member 1 (Tbcld1) to be potently suppressed at both mRNA transcript and protein level with FST treatment (Figure 3 and Supplemental Table 4). Expression analysis in the rAAV:FST-treated 6-month-old mice confirmed suppression of TBC1D1 with a reduction of approximately $80 \%$ in the detected protein compared with the control muscle (Figure 5, D and E). This effect of FST upon TBC1D1 abundance was consistent in the 24-month-old mice treated with rAAV:FST, where TBC1D1 levels were approximately $75 \%$ lower than the control. These results demonstrate that ASB2 regulation by FST is refractory in old mice despite suppression of SMAD2/3 signaling (Figure 1F). The outputs to signaling through this pathway appear to be preserved, as $A s b 2$ mRNA suppression and TBC1D1 downregulation is similar to the results from 6-month-old FST-treated mice.

Having identified a discrepancy in the effects of FST gene delivery upon ASB2 abundance in old versus young mice, we sought to determine the significance of ASB2 as a determinant of FST-mediated muscle hypertrophy by investigating the effects of forced ASB2 expression during FST-mediated muscle growth. We injected the TA muscles of C57B1/6 mice with rAAV:FST plus rAAV:Asb2 (or rAAV:FST plus rAAV:MCS in the contralateral limb). Two and 4 weeks after injection, the muscles were collected and analyzed for changes in mass and muscle fiber morphology (Figure 5F). FST overexpression caused hypertrophy of muscles in both hindlimbs, but muscles that received the coinjection of AAV:Asb2 exhibited an approximately $15 \%$ reduced mass at 2 weeks and an approximately $30 \%$ decrease in mass at 4 weeks compared with muscles receiving rAAV:FST with rAAV:MCS, despite equivalent expression of FST in both conditions (Supplemental Figure 2D). The diminished gains in muscle mass as a consequence of ASB2 overexpression in FST-treated muscles were associated with a consistent effect on the diameter of muscle fibers (Figure 5G). Comparing muscle fibers administered rAAV:FST and rAAV:Asb2 with muscle fibers receiving rAAV:FST identified an approximately $20 \%$ reduction in diameter observed for fibers in the second and third quartile and an approximately $15 \%$ reduction in diameter for fibers in the first quartile. The trend for reduced fiber in muscles given $\mathrm{rAAV}: \mathrm{FST}$ and $\mathrm{rAAV}: \mathrm{Asb} 2$ versus $\mathrm{rAAV}: \mathrm{FST}$ was largely conserved across the entire distribution of muscle fibers (Supplemental Figure 2E). Muscle hypertrophy caused by FST administration is characterized by an increase in protein synthesis (32). To determine if ASB2 expression blunts the FSTstimulated increase in protein synthesis, we measured the global rate of protein synthesis in each condition by surface sensing of translation (SUnSET) (Supplemental Figure 2F) and observed no difference between treatments. Overexpression of ASB2 had a greater negative effect on mass in muscles undergoing FST-induced hypertrophy than muscles administered rAAV:Asb2 alone (Figure 5H). These data demonstrate that $A s b 2$ plays an important role in the regulation of muscle mass and that suppression of $A s b 2$ expression is a critical feature of the cellular events that underlie FST-induced skeletal muscle hypertrophy.

ASB2 expression is responsive to muscle innervation. To investigate whether Asb2 may be regulated in other modes of muscle remodeling, we considered the role of neurologic input. Regulation of muscle mass by E3-ubiquitin ligases is a key factor in the progression of atrophy after denervation, and deterioration of the neuromuscular junction has been identified as a hallmark of sarcopenia associated with aging. Ligases that regulate muscle mass in settings of neurogenic atrophy include tripartite motif-containing 63 (Trim63), F-box protein 32 (Fbxo32), and F-box protein 30 (Fbxo30) (30, 39). On this basis, we sought to evaluate whether ASB2, as another E3 ligase, would be affected after denervation. C57B1/6 mice underwent resection of the peroneal nerve branch in one leg while the contralateral leg underwent the same procedure, but the nerve was left intact for the sham control. We observed temporal variation in ASB2 expression from denervated TA muscle (Figure 5H). Expression of ASB2 was increased 4-fold 3 days after denervation, became suppressed by approximately $75 \% 7$ days after denervation, and eventually resolved to baseline by 28 days after denervation (Figure 5 , I and J). Autophagic activation as a marker of denervation was assessed through expression levels of p62. This demonstrates that Asb2 expression is regulated in muscle after the loss of signaling from the neuromuscular junction, and the response is temporally distinct. 


\section{Discussion}

The seminal discovery of myostatin as a regulator of the TGF- $\beta$ network in skeletal muscle and a negative regulator of muscle growth spawned intense study of TGF- $\beta$ signaling as a therapeutic target in the context of muscle-related disease. Examination of the TGF- $\beta$ network has revealed new roles of other TGF- $\beta$ family ligands as atrophic (activins) and hypertrophic (select BMPs) regulators of muscle attributes. The application of myostatin inhibition in the aged context has shown relatively minor improvements in muscle mass with an appreciable improvement in recovery from muscle injury $(23,40,41)$, although of seemingly lesser magnitude than effects observed during treatment of young mice (42). This may in part be due to the simultaneous elevation of other TGF- $\beta$ family members than can also drive deleterious SMAD2/3 signaling (such as activins) or defects in the hypertrophic response of aged muscles (42). Interventions based around FST may prove more promising because of the potential to inhibit both myostatin and activins, in conjunction with activation of anabolic BMP signaling $(31,32)$. Utilizing rAAV:FST, we discovered that muscle growth due to myostatin/ activin inhibition in aged muscle is achievable but is significantly blunted with advancing age. The capacity for rAAV:FST to suppress myostatin/activin signaling was maintained and points to the differential effect of FST in old muscles being due to differences in the responses of young and old muscle to modulation of SMAD signaling. These observations suggest that interventions intended to conserve or restore muscle mass via modulation of this network may offer useful therapeutic benefits but that the magnitude of effect may diminish as age increases. To understand this observation, we sought to define the changes in gene and protein expression that drive muscle hypertrophy in response to FST treatment. We combined quantitative proteomic and transcriptomic analyses of gene expression in response to FST gene delivery. This analysis identified specific changes in protein abundance and gene expression, including the downregulation of the E3 ubiquitin ligase Asb2.

Suppression of E3 ligases in muscle may be a general strategy FST employs to alter the balance in protein turnover in favor of accretion (32). We found that FST treatment in young adult muscles potently repressed $A s b 2$ transcription and reduced ASB2 protein abundance across all time points during FST expression. Expression of ASB2 resulted in a progressive muscle atrophy that identifies this E3 ligase as an active negative regulator of basal muscle mass. Furthermore, ASB2 repression was required for the maximal muscle hypertrophic response to FST. When expressed in combination with FST, ASB2 resulted in greater than 2-fold relative suppression of muscle mass than ASB2 alone and demonstrated that Asb2 suppression is required for almost a third of FST-induced muscle hypertrophy. This places ASB2 function as being able to modulate muscle mass through a process that is central for the maximal muscle hypertrophy response to FST. By independently inhibiting SMAD2/3 signaling in muscle, we were able to reproduce an equivalent ASB2 downregulation, indicating that within the TGF- $\beta$ network, the SMAD2/3 axis (rather than the SMAD1/5/8 axis) can regulate ASB2 expression. This finding suggests that regulation of basal muscle mass by myostatin/activin signaling would include control of ASB2 function. Interestingly, expression of ASB2 did not change the rate of protein synthesis elicited by FST expression. This suggests that ASB2's target proteins are important mediators of muscle hypertrophy through a mechanism independent of the protein synthesis pathway. Currently, characterized targets of ASB2 include members of the filamin family (filaminA/B), which are critical in the organization and stability of f-actin fibers $(35,36,43)$ and desmin (44). This supports our observation that protein synthesis activation through the mTOR pathway is insufficient to solely account for FST-stimulated muscle hypertrophy (32), and this model incorporates components beyond protein synthesis regulation, of which $A s b 2$ is a critical member.

Progressive deterioration of the interface between muscle fibers and motor nerves culminating in complete denervation is one of the proposed causes of muscle atrophy and frailty observed in sarcopenic muscle $(7,8)$. Nerve resection leads to atrophy in muscle, and the extent to which nerve loss will hamper supportive efforts is unknown (45). We found that ASB2 expression is responsive to nerve input with differential expression observed in a temporal fashion. This is not dissimilar to the expression profile of other E3 ubiquitin ligases that function in the atrophy following denervation (39). This observation broadens the potential role of $A s b 2$ function from TGF- $\beta$ pathway control of muscle mass to also include regulation by innervation and conceivably other conditions in which compromise of the neuromuscular junction is observed. In settings of age-related frailty, changes in Asb2 expression due to compromised nerve input could override suppression in response to FST effects on myostatin/activin. Consistent with this idea, we observed that suppression of Asb2 mRNA by FST treatment is maintained in young and old mice, whereas ASB2 protein levels no longer respond to FST treatment in old mice. This finding indicates that, in the context of aged muscle, Asb2 expression is subject to other mechanisms of regulation, potentially at the posttranscriptional level. 
The combined unbiased proteomic and transcriptomic analyses that we report here has enabled the quantification of global changes in protein expression and gene transcription associated with FST-mediated muscle hypertrophy. This data set highlights the role of Asb2 not only in the regulation of skeletal muscle mass in response to FST treatment, but also as a regulator of basal muscle mass. The absence of ASB2 repression in response to FST treatment is a feature of old, sarcopenic mice. As ASB2 suppression is a condition of maximal FST response, this resistance in old muscle could in part explain the amelioration of muscle growth to myostatin/activin blockade in the aged setting. The proteome/transcriptome data set reported here likely includes components that are unique to TGF- $\beta$ network modulation, but we envisage that there will be common mechanisms shared with other pathways that stimulate muscle hypertrophy. The results of these analyses have highlighted genes and proteins not previously associated with FST-mediated effects in skeletal muscle. Deeper investigation of these FST-responsive genes/proteins could guide strategies to enhance TGF- $\beta$ network manipulation or intervene in these pathways to mimic the changes required for muscle hypertrophy. These data will be a valuable resource for further mechanistic studies of muscle hypertrophy and atrophy via TGF- $\beta$ network regulation and could help identify cellular processes that are critical determinants of muscle attributes in health and disease.

\section{Methods}

Further information can be found in Supplemental Methods.

Generation of $r A A V 6$ vectors. Recombinant AVV were generated as described previously (46). In brief, 10 mg of rAAV6 plasmids containing cDNA constructs were cotransfected with $20 \mathrm{mg}$ of pDGM6 packaging plasmid into HEK293 cells (seeded 16 hours prior at a density of $3.2 \times 10^{6}$ to $3.8 \times 10^{6}$ cells) using the calcium phosphate precipitate method to generate type- 6 pseudo typed viral vectors. After 72 hours, cells and culture medium were collected and homogenized, before clarification, using a $0.22-\mathrm{mm}$ filter (EMD Millipore). Vector was purified by affinity chromatography using a HiTrap heparin column (GE Healthcare), ultracentrifuged overnight, and resuspended in sterile Ringer's solution. Vector concentration was determined using a customized qPCR reaction (Applied Biosystems).

Administration of $r A A V 6$ vector and DOX treatment. Recombinant viral vector stocks were used at dosage of $1 \times 10^{9}$ to $5 \times 10^{10}$ vectors genomes as indicated. AAV:indFST at $1 \times 10^{9}, \mathrm{AAV}$ :FST at $1 \times 10^{9}$, AAV:TetON at $5 \times 10^{9}, \mathrm{AAV}: \mathrm{caALK} 3$ at $1 \times 10^{10}, \mathrm{AAV}: \mathrm{SMAD} 7$ at $1 \times 10^{10}, \mathrm{AAV}: \mathrm{Asb} 2$ at $5 \times 10^{9}$, and $\mathrm{AAV}: \mathrm{MCS}$ at a matched dosage to the tested AAV. i.m. injections of rAAV6 vector were administered to mice at the indicated age or otherwise were performed in 8- to 10 -week-old male C57BL/6 mice as described previously (Alfred Medical Research \& Education Precinct) (32). The left TA (LTA) muscle received an i.m. injection of indicated rAAV6 vector serving as the control sample while the right TA (RTA) muscle was injected with indicated rAAV6 vectors serving as the treatment sample. For inducible expression, mice were housed for 7 days after injection of rAAV6 vectors and then placed on a standard chow diet containing $600 \mathrm{mg} / \mathrm{kg}$ DOX (Specialty Feeds) for indicated durations.

Transcriptome sequencing and bioinformatics. Whole transcriptome sequencing (mRNA-Seq) was completed as described previously (47). Briefly, TA muscles injected with AAV:MCS or AAV:FST were used to generate cDNA libraries from poly A+ purified mRNA samples according to the manufacturer's instructions (TruSeq RNA sample preparation kit, Illumina). Transcriptome libraries were sequenced on an Illumina GAIIx instrument, with the resulting $36 \mathrm{bp}$ single-end reads mapped to the mouse genome (mm10 assembly) using TopHat (48). Gene transcript levels were determined via Cuffdiff in the form of FPKM (RPKM) values by correcting for multireads and using geometric normalization (49). GO analyses of molecular function were analyzed using the bioinformatics resource DAVID (National Institute of Allergy and Infectious Diseases, NIH) $(50,51)$. The sequencing data are deposited with Gene Expression Omnibus (GEO) under the accession number GSE78965.

Proteomic analysis, mass spectrometry, and bioinformatics. Protein extracted from TA muscles was digested with LysC and trypsin and desalted with tC18 solid phase extraction columns. Peptides were labeled with iTRAQ according to the manufacturer's instructions and fractionated by hydrophilic interaction liquid chromatograph (HILIC) into 12 fractions. Each fraction was analyzed by liquid chromatography/tandem mass spectrometry using data-dependent acquisition (Q-Exactive, Thermo Fisher). All statistical analysis was performed in the R programming environment (http://www.r-project.org). Protein quantification was performed using the median values of all PSMs of the protein group and normalized within each condition using median absolute deviation for each of the 3 treatments. Moderated 
1 -sample $t$ tests were performed at each time point on the $\log _{2}$ transformed ratios relative to no-DOX control to identify significantly regulated proteins. In order to identify proteins that were altered at 2 and 28 days, we performed a 2-dimensional hypothesis test using DPA and OSP method for combining $P$ values across the 2 treatments and transformed the $t$ statics to Z-scores. Hypotheses testing for distinct responses across the time points in the following directions $(-1,-1)$ and $(1,1)$, where -1 represents downregulation and 1 represents upregulation, was performed to identify proteins that were concordantly up- or downregulated at 2 or 28 days. Significance of each protein was defined as combined $P<0.001$. Permutation test-based methods such as the GSEA $(52,53)$ were performed to identify biological pathways that were either significantly up-, down-, or mixed-regulated at each time point/treatment $(P<0.05)$. The gene sets or pathways tested for differential expression in our proteomics data was obtained from the Molecular Signature Database (MSigDB; http://software.broadinstitute.org/gsea/msigdb) collection. The union of significantly altered pathways at least at one time point is represented. KEGG-annotated disease pathways were eliminated from this analysis.

Integrated analysis of proteomic and transcriptomic data. We defined genes that were significantly altered $(P<0.05$ from adjusted $t$ tests, FDR $=0.05)$ at 3 days as acute responders and genes that were altered at 7 and 14 days as chronic responders in the transcriptomics data. In order to represent the transcriptome versus proteome changes in a scatterplot, we calculated an integrated $P$ value for each gene in the common data set from the transcriptomic data analysis. We used Fisher's combined probability test to combine the $P$ values from the independent differential expression analysis at 3,7 , and 14 days for every gene in the transcriptomics data. The combined $P$ value of every protein from the proteomic analysis was calculated previously using DPA (54) as explained above. We transformed these integrated $P$ values in the negative $\log _{10}$ scale for each data set and generated a scatterplot for the common data set with the proteomics data-derived combined $P$ value in the $x$ axis and the transcriptomics data-derived Fisher's combined $P$ value in the $y$ axis.

Statistics. For statistical analysis not related to the proteomic and RNA-Seq data sets, data are presented as mean \pm SEM, and biological replicates are stated in Figure legends. No animals were excluded from statistical analysis. One-way ANOVA tests were used to assess statistical differences across multiple conditions, with the Bonferroni post hoc test used for comparisons between the specific group means. Comparisons between 2 conditions used the 1-way or paired Student's $t$ test as indicated in Figure legends. All differences reported are $P<0.05$.

Study approval. All experiments were conducted in accordance with the relevant codes of practice for the care and use of animals for scientific purposes (NIH, 1985; the National Health and Medical Council of Australia, 2004). All animal work performed in this study was conducted with approval from the Alfred Medical Research and Education Precinct Animal Ethics Committee (AMREP AEC) (Melbourne, Australia).

\section{Author contributions}

JRD, KIW, and PG designed the experiments. JRD, KIW, BLP, RC, JGR, LC, and HQ undertook the experimental work. LC, HQ, VS, JC, MS, DEJ, and PG contributed reagents and hardware resources. JRD, BLP, RC, JGR, and PG analyzed the data. JRD, BLP, RC, JGR, and PG wrote the manuscript. All authors reviewed and edited the manuscript.

\section{Acknowledgments}

The Authors thank G. Gutierrez-Cruz (NIAMS, NIH) for assistance with preparing and processing of muscle samples for gene expression profiling. This research was supported by funding from the University of Washington's Nathan Shock Center of Excellence in the Basic Biology of Aging, the National Health and Medical Research Council of Australia (NHMRC, grant 526648), the Thomas Baker Family Foundation (awarded to P. Gregorevic), the National Institute of Aging at the NIH (5R01AG033610-03, awarded to J. Chamberlain), and in part by the Intramural Research Program of NIAMS at the NIH (awarded to V. Sartorelli). B.L. Parker is supported by an NHMRC Early Career Fellowship. D.E. James is supported by an NHMRC Senior Principal Research Fellowship. P. Gregorevic is supported by a NHMRC Career Development Fellowship (1046782). The Baker IDI Heart \& Diabetes Institute is supported in part by the Operational Infrastructure Support Program of the Victorian Government, Australia. The contents of the published material are solely the responsibility of the individual authors and the administering institution and do not reflect the views of the funders of this work. 
Address correspondence to: Paul Gregorevic, Baker IDI Heart and Diabetes Institute, P.O. Box 6492, Melbourne, VIC 3004, Australia. Phone: 61.3.8532.1224; E-mail: Paul.Gregorevic@BakerIDI.edu.au.

1. Doherty TJ. Invited Review: Aging and sarcopenia. J Appl Physiol. 2003;95(4):1717-1727.

2. van Kan GA. Epidemiology and consequences of sarcopenia. J Nutr Health Aging. 2009;13(8):708-712.

3. Orentreich N, Brind JL, Vogelman JH, Andres R, Baldwin H. Longitudinal measurements of plasma dehydroepiandrosterone sulfate in normal men. J Clin Endocrinol Metab. 1992;75(4):1002-1004.

4. Khosla S, Melton LJ, Atkinson EJ, O'Fallon WM, Klee GG, Riggs BL. Relationship of serum sex steroid levels and bone turnover markers with bone mineral density in men and women: A key role for bioavailable estrogen. J Clin Endocrinol Metab. 1998;83(7):2266-2274

5. Altun M, et al. Muscle wasting in aged, sarcopenic rats is associated with enhanced activity of the ubiquitin proteasome pathway. J Biol Chem. 2010;285(51):39597-39608.

6. Ibebunjo C, et al. Genomic and proteomic profiling reveals reduced mitochondrial function and disruption of the neuromuscular junction driving rat sarcopenia. Mol Cell Biol. 2013;33(2):194-212.

7. Deschenes MR, Roby MA, Eason MK, Harris MB. Remodeling of the neuromuscular junction precedes sarcopenia related alterations in myofibers. Exp Gerontol. 2010;45(5):389-393.

8. Chai RJ, Vukovic J, Dunlop S, Grounds MD, Shavlakadze T. Striking denervation of neuromuscular junctions without lumbar motoneuron loss in geriatric mouse muscle. PLoS One. 2011;6(12):e28090.

9. von Haehling S, Morley JE, Anker SD. An overview of sarcopenia: facts and numbers on prevalence and clinical impact. $J$ Cachexia Sarcopenia Muscle. 2010;1(2):129-133.

10. Cohen S, Nathan JA, Goldberg AL. Muscle wasting in disease: molecular mechanisms and promising therapies. Nat Rev Drug Discov. 2015;14(1):58-74.

11. McPherron AC, Lawler AM, Lee S-J. Regulation of skeletal muscle mass in mice by a new TGF- $\beta$ superfamily member. Nature. 1997;387(6628):83-89.

12. Lee SJ, McPherron AC. Regulation of myostatin activity and muscle growth. Proc Natl Acad Sci U S A. 2001;98(16):9306-9311.

13. Welle S, Bhatt K, Pinkert CA, Tawil R, Thornton CA. Muscle growth after postdevelopmental myostatin gene knockout. $A m J$ Physiol Endocrinol Metab. 2007;292(4):E985-EE91.

14. Whittemore LA, et al. Inhibition of myostatin in adult mice increases skeletal muscle mass and strength. Biochem Biophys Res Commun. 2003;300(4):965-971.

15. Zimmers TA, et al. Induction of cachexia in mice by systemically administered myostatin. Science. 2002;296(5572):1486-1488

16. Chen JL, et al. Development of novel activin-targeted therapeutics. Mol Ther. 2015;23(3):434-444.

17. Zhou X, et al. Reversal of cancer cachexia and muscle wasting by ActRIIB antagonism leads to prolonged survival. Cell. 2010;142(4):531-543.

18. Chen JL, et al. Elevated expression of activins promotes muscle wasting and cachexia. FASEB J. 2014;28(4):1711-1723.

19. Matzuk MM, Finegold MJ, Mather JP, Krummen L, Lu H, Bradley A. Development of cancer cachexia-like syndrome and adrenal tumors in inhibin-deficient mice. Proc Natl Acad Sci U S A. 1994;91(19):8817-8821.

20. Loumaye A, et al. Role of Activin A and myostatin in human cancer cachexia. J Clin Endocrinol Metab. 2015;100(5):2030-2038.

21. Baccarelli A, et al. Activin A serum levels and aging of the pituitary-gonadal axis: a cross-sectional study in middle-aged and elderly healthy subjects. Exp Gerontol. 2001;36(8):1403-1412.

22. Lach-Trifilieff E, et al. An antibody blocking activin type II receptors induces strong skeletal muscle hypertrophy and protects from atrophy. Mol Cell Biol. 2014;34(4):606-618.

23. Becker C, et al. Myostatin antibody (LY2495655) in older weak fallers: a proof-of-concept, randomised, phase 2 trial. Lancet Diabetes Endocrinol. 2015;3(12):948-957.

24. Mendell JR, et al. A phase 1/2a follistatin gene therapy trial for becker muscular dystrophy. Mol Ther. 2015;23(1):192-201

25. Thompson TB, Lerch TF, Cook RW, Woodruff TK, Jardetzky TS. The structure of the follistatin:activin complex reveals antagonism of both type I and type II receptor binding. Dev Cell. 2005;9(4):535-543.

26. Harrington AE, Morris-Triggs SA, Ruotolo BT, Robinson CV, Ohnuma S, Hyvönen M. Structural basis for the inhibition of activin signalling by follistatin. EMBO J. 2006;25(5):1035-1045

27. Mather JP, Roberts PE, Krummen LA. Follistatin modulates activin activity in a cell- and tissue-specific manner. Endocrinology. 1993;132(6):2732-2734.

28. Matzuk MM, Lu N, Vogel H, Sellheyer K, Roop DR, Bradley A. Multiple defects and perinatal death in mice deficient in follistatin. Nature. 1995;374(6520):360-363.

29. Miller TM, et al. Gene transfer demonstrates that muscle is not a primary target for non-cell-autonomous toxicity in familial amyotrophic lateral sclerosis. Proc Natl Acad Sci U S A. 2006;103(51):19546-19551.

30. Sartori R, et al. BMP signaling controls muscle mass. Nat Genet. 2013;45(11):1309-1318.

31. Winbanks CE, et al. The bone morphogenetic protein axis is a positive regulator of skeletal muscle mass. J Cell Biol. 2013;203(2):345-357.

32. Winbanks CE, et al. Follistatin-mediated skeletal muscle hypertrophy is regulated by Smad3 mTOR independently of myostatin. J Cell Biol. 2012;197(7):997-1008.

33. Guibal FC, et al. ASB-2 inhibits growth and promotes commitment in myeloid leukemia cells. J Biol Chem. 2002;277(1):218-224.

34. Heuzé ML, et al. ASB2 is an Elongin BC-interacting protein that can assemble with Cullin 5 and Rbx1 to reconstitute an E3 ubiquitin ligase complex. J Biol Chem. 2005;280(7):5468-5474.

35. Bello NF, et al. The E3 ubiquitin ligase specificity subunit ASB2 $\beta$ is a novel regulator of muscle differentiation that targets filamin B to proteasomal degradation. Cell Death Differ. 2009;16(6):921-932.

36. Razinia Z, Baldassarre M, Cantelli G, Calderwood DA. ASB2 $\alpha$, an E3 ubiquitin ligase specificity subunit, regulates cell spreading and triggers proteasomal degradation of filamins by targeting the filamin calponin homology 1 domain. $J$ Biol Chem. 
2013;288(44):32093-32105.

37. Hayashi $\mathrm{H}$, et al. The MAD-related protein Smad7 associates with the TGF $\beta$ receptor and functions as an antagonist of TGF $\beta$ signaling. Cell. 1997;89(7):1165-1173.

38. Hanyu A, Ishidou Y, Ebisawa T, Shimanuki T, Imamura T, Miyazono K. The N domain of Smad7 is essential for specific inhibition of transforming growth factor- $\beta$ signaling. J Cell Biol. 2001;155(6):1017-1028.

39. Bodine SC, et al. Identification of ubiquitin ligases required for skeletal muscle atrophy. Science. 2001;294(5547):1704-1708.

40. LeBrasseur NK, Schelhorn TM, Bernardo BL, Cosgrove PG, Loria PM, Brown TA. Myostatin inhibition enhances the effects of exercise on performance and metabolic outcomes in aged mice. J Gerontol A Biol Sci Med Sci. 2009;64A(9):940-948.

41. Murphy KT, et al. Antibody-directed myostatin inhibition in 21-mo-old mice reveals novel roles for myostatin signaling in skeletal muscle structure and function. FASEB J. 2010;24(11):4433-4442.

42. Murphy KT, et al. Antibody-directed myostatin inhibition improves diaphragm pathology in young but not adult dystrophic mdx mice. Am J Pathol. 2010;176(5):2425-2434.

43. Heuzé ML, et al. ASB2 targets filamins A and B to proteasomal degradation. Blood. 2008;112(13):5130-5140.

44. Thottakara T, et al. The E3 ubiquitin ligase Asb2 $\beta$ is downregulated in a mouse model of hypertrophic cardiomyopathy targets desmin for proteasomal degradation. J Mol Cell Cardiol. 2015;87:214-224.

45. Quy PN, Kuma A, Pierre P, Mizushima N. Proteasome-dependent activation of mammalian target of rapamycin complex 1 (mTORC1) is essential for autophagy suppression and muscle remodeling following denervation. J Biol Chem. 2013;288(2):1125-1134.

46. Gregorevic P, et al. Systemic delivery of genes to striated muscles using adeno-associated viral vectors. Nat Med. 2004;10(8):828-834

47. Ryall James G, et al. The NAD ${ }^{+}$-dependent SIRT1 deacetylase translates a metabolic switch into regulatory epigenetics in skeletal muscle stem cells. Cell Stem Cell. 2015;16(2):171-183.

48. Trapnell C, Pachter L, Salzberg SL. TopHat: discovering splice junctions with RNA-Seq. Bioinformatics. 2009;25(9):1105-1111.

49. Trapnell C, Hendrickson DG, Sauvageau M, Goff L, Rinn JL, Pachter L. Differential analysis of gene regulation at transcript resolution with RNA-seq. Nat Biotechnol. 2013;31(1):46-53.

50. Huang DW, Sherman BT, Lempicki RA. Systematic and integrative analysis of large gene lists using DAVID bioinformatics resources. Nat Protoc. 2008;4(1):44-57.

51. Huang DW, Sherman BT, Lempicki RA. Bioinformatics enrichment tools: paths toward the comprehensive functional analysis of large gene lists. Nucleic Acids Res. 2009;37(1):1-13.

52. Subramanian A, et al. Gene set enrichment analysis: A knowledge-based approach for interpreting genome-wide expression profiles. Proc Natl Acad Sci U S A. 2005;102(43):15545-15550.

53. Smyth GK. Linear models and empirical bayes methods for assessing differential expression in microarray experiments. Stat Appl Genet Mol Biol. 2004;3:Article3.

54. Yang P, et al. Direction pathway analysis of large-scale proteomics data reveals novel features of the insulin action pathway. Bioinformatics. 2014;30(6):808-814. 